\title{
TCP10L synergizes with MAD1 in transcriptional suppression and cell cycle arrest through mutual interaction
}

\author{
Suqin Shen ${ }^{1}$, Jie Zuo ${ }^{1}$, Huan Feng ${ }^{1}$, Meirong Bai ${ }^{2}$, Chenji Wang ${ }^{1}$, Youheng Wei ${ }^{1}$, Yanhong $L^{1}{ }^{1}$, Yichen $L e^{1}$, Jiaxue Wu ${ }^{1}$, \\ Yanhua $\mathrm{Wu}^{1, *} \mathcal{E}$ Long $\mathrm{Yu}^{1, *}$ \\ ${ }^{1}$ State Key Laboratory of Genetic Engineering, Institute of Genetics, School of Life Sciences, Fudan University, Shanghai 200433, P. R. \\ China, ${ }^{2}$ Cardiovascular Research Institute and Department of Physiology, University of California, San Francisco, San Francisco, CA 94158 , \\ USA
}

T-complex protein 10A homolog 2 (TCP10L) was previously demonstrated to be a potential tumor suppressor in human hepatocellular carcinoma (HCC). However, little is known about the molecular mechanism. MAX dimerization protein 1 (MAD1) is a key transcription suppressor that is involved in regulating cell cycle progression and Myc-mediated cell transformation. In this study, we identified MAD1 as a novel TCP10L-interacting protein. The interaction depends on the leucine zipper domain of both TCP10L and MAD1. TCP10L, but not the interaction-deficient TCP10L mutant, synergizes with MAD1 in transcriptional repression, cell cycle G1 arrest and cell growth suppression. Mechanistic exploration further revealed that TCP10L is able to stabilize intracellular MAD1 protein level. Consistently, the MAD1-interaction-deficient TCP10L mutant exerts no effect on stabilizing the MAD1 protein. Taken together, our results strongly indicate that TCP10L stabilizes MAD1 protein level through direct interaction, and they cooperatively regulate cell cycle progression. [BMB Reports 2016; 49(6): 325-330]

\section{INTRODUCTION}

The MYC/MAX/MAD family, a group of transcription factors containing basic helix-loop-helix leucine-zipper (bHLHZip) domains, plays a vital role in the control of cell physiology. The family members mainly act as molecular switches, in that they

*Corresponding authors. Yanhua Wu, Tel: +86-21-51630593; Fax: +86-21-51630567; E-mail: yanhuawu@fudan.edu.cn, Long Yu, Tel: +86-21-51630580; Fax: +86-21-51630567; E-mail: longyu@fudan. edu.cn.

http://dx.doi.org/10.5483/BMBRep.2016.49.6.248

Received 30 November 2015, Revised 23 December 2015, Accepted 23 December 2015

Keywords: Hepatocellular carcinoma, MAX dimerization protein 1, Protein degradation, Protein-protein interaction, T-complex protein $10 \mathrm{~A}$ homolog 2 can either transcriptionally activate or repress target gene expression through forming different transcriptional complexes (1). MYC forms a heterodimer with MAX, and then the MYC/MAX complex specifically binds to "E-box" (consensus sequence: $5^{\prime}$-CACGTG-3') elements located on the promoter region of target genes, leading to the activation of gene transcription (2). MYC expression is able to stimulate the G1-S phase transition of the cell cycle and induce cell transformation (3). Another family member, MAX dimerization protein 1 (MAD1), is able to compete with MYC to form a heterodimer with MAX, and MAD1/MAX complex usually binds to the same "E-box" sequences upstream of target genes as MYC/MAX (4). However, MAD1 functions as an antagonist of MYC (5). The switch of MYC/MAX to MAD1/MAX usually turns gene transcription off, due to the recruitment of histone deacetylases such as mSIN3 to DNA, as well as the stimulation of chromatin conformation changes (6). Consistent with its gene transcriptional activity, MAD1 is able to inhibit the cell cycle $\mathrm{G} 1 / \mathrm{S}$ progression, and suppress myc-mediated cell transformation (1).

T-complex protein 10A homolog 2 (TCP10L), mapped to chromosome 21q22.11, was first cloned by Chen Z in 2003 through a differentially displayed hybrid from a human liver cDNA library (7). TCP10L contains a putative leucine zipper (LZ) domain, which is required for its homo-dimerization but not for its nuclear localization (8). Recently, our lab reported that TCP10L was a potential tumor suppressor in hepatocellular carcinoma (HCC) (9). In this study, we aim to further explore the molecular mechanism accounting for TCP10L's function in HCC cells. We characterized MAD1 as a novel TCP10L-interacting protein. The key domain required for the interaction was identified through constructing different deletion mutants. Moreover, the functional effect of TCP10L-MAD1 interaction was also studied.

\section{RESULTS}

\section{TCP10L directly interacts with MAD1}

Our lab has previously identified MAD4 as a TCP10L-interacting protein through yeast two hybrid methods (10). Both 
MAD1 and MAD4 belong to the MAD protein family, and they share similar domain architecture. We asked whether TCP10L could also interact with MAD1 and further affect MAD1's function. To address this question, we first carried out co-immunoprecipitation (co-IP) assays. As shown in Fig. 1A, HA-TCP10L was immunoprecipitated by Myc-MAD1 suggesting an interaction between these two proteins. Similar results were also obtained in the reciprocal co-IP experiment in which Myc-TCP10L was able to immunoprecipitate HA-MAD1 (Fig. 1B). Next, we extended our analysis by investigating whether endogenous TCP10L and MAD1 can associate with each other. In this case, SMMC-7721 cells were pretreated with the protease inhibitor MG132. As shown in Fig. 1C, endogenous TCP10L was also efficiently immunoprecipitated by the anti-MAD1 antibody but not the IgG control. To further confirm the interaction relationship between TCP10L and MAD1, immunofluorescence-based cell staining was performed. As shown in Fig. 1D, GFP-tagged TCP10L was diffusely localized in the nucleus, which is consistent with its role in gene transcription. By contrast, MAD1 was localized in a specific speckled pattern in the nucleus, which is consistent with a previous report (11). Interestingly, when TCP10L and MAD1 were co-expressed in the same cells, TCP10L was not only co-localized with MAD1 but also displayed a speckled pattern in

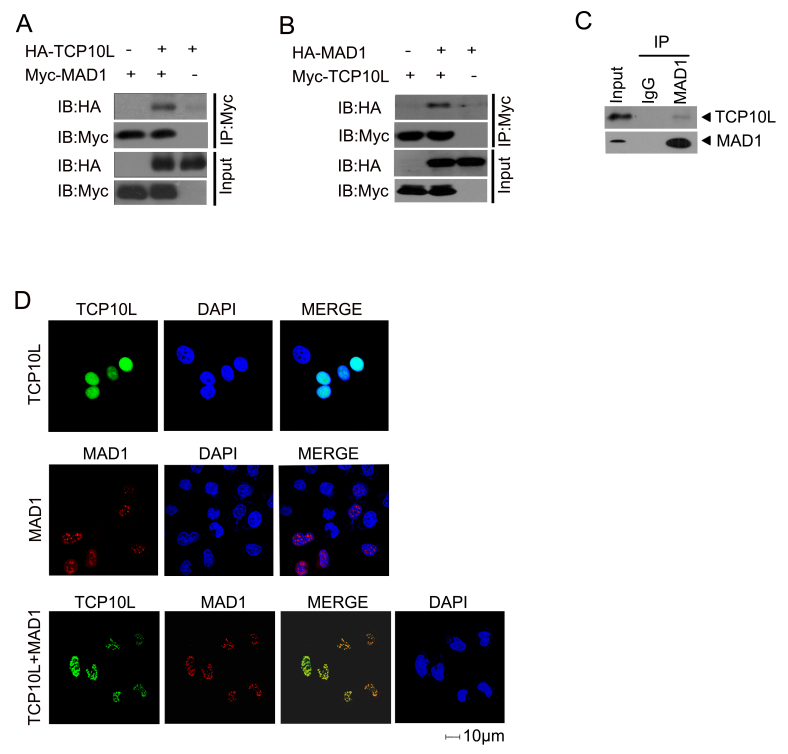

Fig. 1. TCP10L interacts with MAD1 in vitro and in vivo. (A) Co-IP assay was performed in HEK293T cells ectopically expressing HA-MAD1 and Myc-TCP10L with an anti-Myc antibody. (B) Co-IP assay was performed in HEK293T cells ectopically expressing Myc-MAD1 and HA-TCP10L with an anti-Myc antibody. (C) Endogenous association between MAD1 and TCP10L was performed in SMMC-7721 cells using an anti-MAD1 antibody. (D) Immunofluorescence staining of HeLa cells ectopically expressing indicated recombinant plasmids. Cells were immunostained with anti-HA antibody, and counterstained by DAPI before visualization. the nucleus. The change of the localization pattern of TCP10L preliminarily indicates that once TCP10L and MAD1 were associated, TCP10L might be involved in the physiological function of MAD1.

\section{The leucine zipper domains are essential for the TCP10 L-MAD1 interaction}

To understand the structural requirements for the interaction between TCP10L and MAD1, a deletion mutant approach was utilized to map the essential domains. There is a typical leucine zipper domain (LZ) consisting of a tetrad repeat of leucine residues located in the N-terminal domain of TCP10L. Thus, wild-

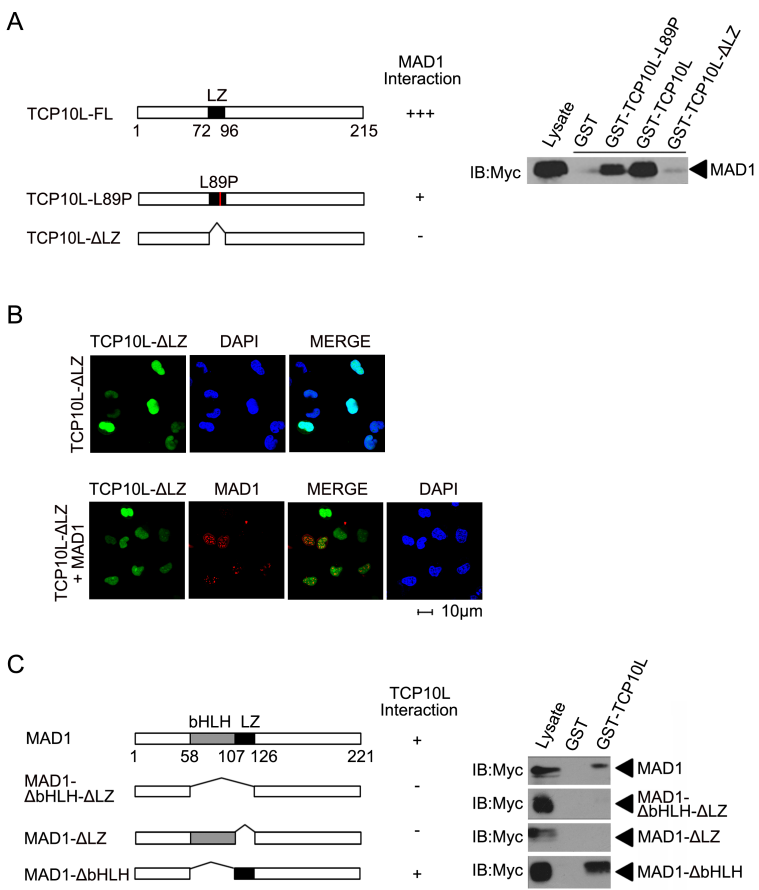

Fig. 2. The leucine zipper domains of both TCP10L and MAD1 are required for the TCP10L-MAD1 interaction. (A) Left panel: Schematic representation of full length TCP10L protein and mutants. Right panel: Binding capacity of TCP10L to MAD1 is indicated with the symbol. Bacterially expressed GST fusion proteins of TCP10L-FL, TCP10L-L89P and TCP10L- $\Delta$ LZ mutants were bound to glutathione-Sepharose beads, respectively, then incubated with lysates of HEK293T overexpressing Myc-MAD1. Bound Myc-MAD1 was subjected to Western blot with anti-Myc antibody. (B) Left panel: Schematic representation of full length MAD1 protein and the deletion mutants. Binding capacity of MAD1 to TCP10L is indicated with the symbol. Right panel: Bacterially expressed GST fusion proteins of TCP10L protein was bound to glutathioneSepharose beads, then incubated with lysates of HEK293T overexpressing full length MAD1 and its deletion mutant, respectively. Bound MAD1 was subjected to western blot with an anti-Myc antibody. (C) Immunofluorescence staining of HeLa cells ectopically expressing TCP10L- $\Delta \mathrm{LZ}$ mutants alone or together with MAD1. Cells were immunostained with anti-HA antibody, and counterstained by DAPI before visualization. 
type TCP10L and two TCP10L mutants, TCP10L-L89P (amino acid 89, Leu to Pro) and TCP10L- $\Delta \mathrm{LZ}$ (deletion of the whole LZ domain), were constructed in a GST-tagged prokaryotic expression vector pGX-4T-2 (Fig. 2A, left panel) to perform a GST-pull down assay. As shown in the right panel of Fig. 2A, GST-TCP10L protein can directly bind to the Myc-MAD1 protein expressed in HEK293T cells. However, deletion of the LZ domain of TCP10L totally abolished the interaction, whereas L89P mutation displayed reduced interaction with MAD1. Next, immunofluorescence staining was performed in cells transfected with TCP10L- $\Delta \mathrm{LZ}$ alone and cells co-transfected with TCP10L- $\Delta \mathrm{LZ}$ and MAD1. As shown in Fig. 2B, GFP-tagged TCP10L- $\Delta \mathrm{LZ}$ was also diffusely localized in the nucleus, suggesting that the LZ domain of TCP10L is not required for nuclear import. However, while TCP10L- $\Delta \mathrm{LZ}$ and MAD1 were co-expressed in the same cells, no obvious co-localization pattern was found. MAD1 was localized in a speckled pattern in the nucleus, but TCP10L- $\Delta \mathrm{LZ}$ remained in a diffused pattern.

We next addressed the question of which domain of MAD1 contributes to the TCP10L-MAD1 interaction. Three MAD1 mutants, MAD1 lacking the bHLH domain (MAD1- $\triangle b H L H)$, MAD1 lacking the $\mathrm{LZ}$ domain (MAD1- $\triangle \mathrm{LZ}$ ) or MAD1 lacking both the bHLH and $\mathrm{LZ}$ domains (MAD1- $\Delta \mathrm{bHLH}-\Delta \mathrm{LZ}$ ), were constructed (Fig. 2B, left panel). As shown in the right panel of Fig. 2B, GST-TCP10L showed a strong interaction with either MAD1 or the MAD1- $\Delta b H L H$ mutant but not MAD1- $\Delta L Z$ or $M A D 1-\Delta b H L H-\Delta L Z$, suggesting that the intact $L Z$ domain of MAD1 is also specifically required for the interaction between TCP10L and MAD1.

\section{TCP10L synergizes with MAD1 in regulating gene transcription and cell cycle progression}

When MAD1 was found to be able to change the subcellular localization of TCP10L, we raised a hypothesis that TCP10L might be involved in the MAD1 pathway, acting as a modulator of MAD1. To demonstrate our hypothesis, gene transcriptional activity of TCP10L was first assessed. In a dual luciferase assay, pGL-myc-Luc, an artificial c-myc promoter composed of four tandem E-box repeats upstream of a luciferase reporter gene was co-transfected with plasmids of interest. As shown in Fig. 3A, overexpression of MAD1 markedly repressed the transcriptional activity of the reporter gene, which is consistent with others' reports (12). Interestingly, TCP10L, but not the TCP10L- $\Delta \mathrm{LZ}$ mutant, exerted a repressive role on the reporter gene transcriptional activity. Moreover, co-expression of TCP10L, but not the TCP10L- $\Delta \mathrm{LZ}$ mutant with MAD1 achieved a synergized suppression effect $(63.2 \% \pm 2.81 \%$ vs. $21.24 \% \pm 3.02 \%$ ). To confirm this finding, the mRNA expression of two target genes under control of MAD1 (human telomerase reverse transcriptase, $h T E R T$ and ornithine decarboxylase, ODC), was investigated through qRT-PCR experiments. As shown in Fig. 3B and 3C, over-expression of either MAD1 or TCP10L led to reduced mRNA levels of hTERT and ODC. Meanwhile, co-expression of TCP10L, but not the
TCP10L- $\Delta$ LZ mutant with MAD1 achieved maximal reduction of mRNA levels of both $h T E R T$ and ODC.

We further investigated the functional relationship between TCP10L and MAD1 in regulating cell cycle progression. As expected, G1 arrest was not only observed in cells expressing MAD1 but also in cells expressing TCP10L (Fig. 3D and 3E). More importantly, co-expression of TCP10L, but not the TCP10L- $\Delta \mathrm{LZ}$ mutant, remarkably enhanced MAD1-mediated G1 arrest $(47.24 \% \pm 1.62 \%$ vs. $58.89 \% \pm 2.57 \%)$. Consistently, in an in vitro cell growth assay, overexpression of TCP10L or MAD1 alone suppressed SMMC-7721 cell growth, and co-expression of TCP10L, but not the TCP10L- $\Delta$ LZ mutant amplified the suppressive effect of MAD1 (Fig. 3F). Taken together, these results strongly suggest that $\mathrm{TCP} 10 \mathrm{~L}$ can synergize with MAD1 in suppressing target gene expression, inducing G1 arrest and regulating cell growth.
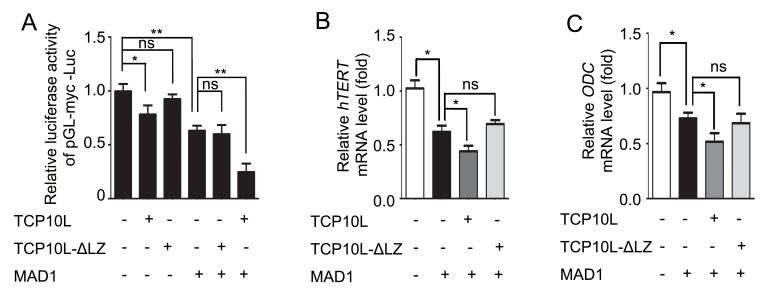

D

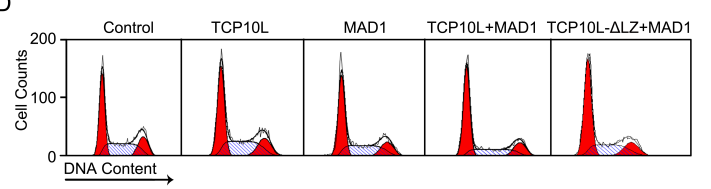

E
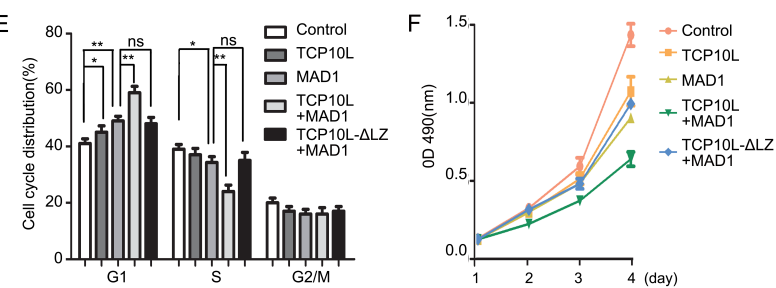

Fig. 3. TCP10L synergizes with MAD1 in suppressing gene transcription and cell cycle progression. (A) The luciferase activities of c-Myc-Luc upon TCP10L and/or MAD1 expression were measured by luminometer in HEK293T cells at $30 \mathrm{~h}$ post transfection. (B, C) The mRNA levels of MAD1 target genes hTERT (B) or ODC (C) was detected using qRT-PCR methods in HEK293T cells transfected with indicated plasmids. The mean values $( \pm$ S.D.) are shown. (D, E) Cell cycle distributions were analyzed by FACS in SMMC-7721 cells transfected with indicated plasmids. Representative pictures were presented in (D) and statistical data were shown in (E). (F) Cell growth curve of SMMC-7721 transfected with indicated plasmids was calculated by MTS assay. The mean values $( \pm$ S.D.) are shown 


\section{TCP10L stabilizes MAD1 by preventing its protein degradation}

After revealing the synergistic effect of TCP10L and MAD1 in control of gene expression and cell cycle, another remaining question is that why the interaction between TCP10L and MAD1 contributes to enhanced activity of MAD1? It has been reported that endogenous MAD1 protein in cancer cells usually has a very short half-life (15-30 min) (13). So we carried out experiments to investigate the effect of TCP10L on MAD1 protein stability. HEK293T cells were co-transfected with HA-MAD1 and increasing amounts of Myc-TCP10L. As shown in Fig. 4A, although same amount of MAD1 plasmids were transfected in each group, the MAD1 protein level was remarkably increased when TCP10L was co-expressed in a dose-dependent manner. In contrast, no obvious change of MAD1 protein was shown in cells co-transfected with Myc-TCP10L- $\Delta$ LZ (Fig. 4B).

Next, two specific siRNAs were designed to knock down endogenous TCP10L. As shown in Fig. 4C, knockdown of

A

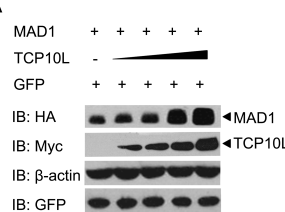

B
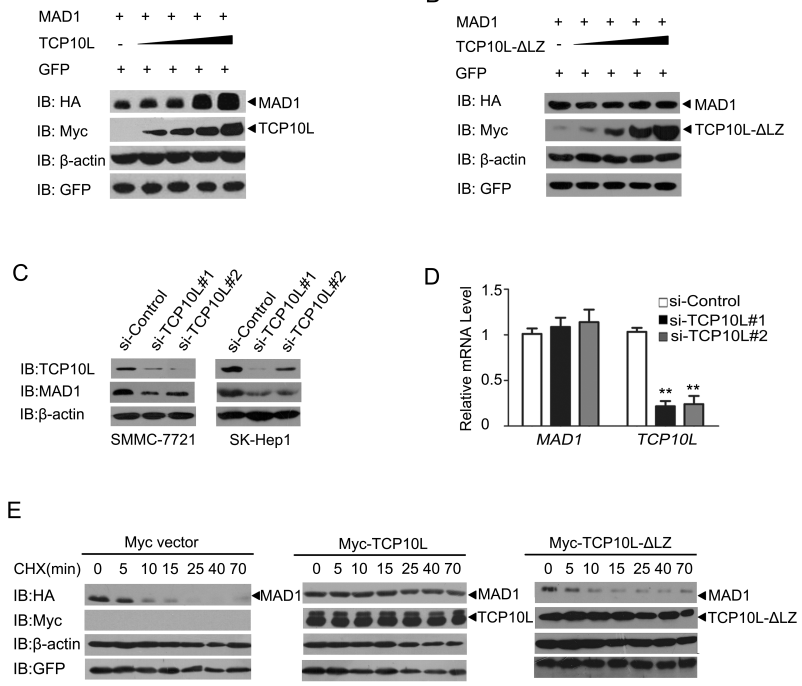

Fig. 4. TCP10L stabilizes MAD1 by preventing its protein degradation. (A, B) MAD1 and increasing amounts of full length TCP10L (A) or TCP10L- $\Delta \mathrm{LZ}$ mutant (B) were co-transfected into HEK293T cells and the protein level of MAD1 was detected by Western blot analysis. (C) SMMC-7721 and SK-Hep1 cells were transfected with siRNA against TCP10L (si-TCP10L\#1, si-TCP10L\#2) and control siRNA. The protein level of MAD1 and TCP10L were detected by Western blot analysis. (D) The mRNA level of TCP1OL and MAD1 in SMMC-7721 cells transfected with indicated siRNAs was measured by qRT-PCR. The mRNA level of GAPDH was used for normalization. The mean values $( \pm$ S.D.) are shown. (E) $\mathrm{CHX}$ chase assay of MAD1 upon TCP10L expression. HEK293T cells were co-transfected with HA-MAD1, along with empty vector, or Myc-TCP10L, or Myc-TCP10L- $\Delta \mathrm{LZ}$ mutant before being treated with $\mathrm{CHX}$. Cell lysates were subjected to Western blot. The pEGFP-N1 expression was included as a transfection efficiency control.
TCP10L in both cell lines resulted in an obvious decrease in the level of endogenous MAD1. To exclude that TCP10L might affect MAD1 gene transcription, a qRT-PCR assay was performed and no statistical difference in MAD1 mRNA levels was shown in cells expressing TCP10L in comparison with control cells (Fig. 4D). We further performed a cycloheximide (CHX) chase assay to examine the effect of TCP10L on MAD1 protein turnover. HEK293T cells were transfected with HA-MAD1 and TCP10L or TCP10L- $\triangle \mathrm{LZ}$ mutant before treatment with $\mathrm{CHX}$. As shown in Fig. 4E, co-expression of TCP10L and MAD1 resulted in a striking prolonged half-life of MAD1. In contrast, neither the empty vector nor TCP10L- $\Delta \mathrm{LZ}$ mutant displayed the similar function. Taken together, the TCP10LMAD1 interaction could promote MAD1 stabilization, which might contribute to the synergistic effect on cell events.

\section{DISCUSSION}

In this study, we characterize MAD1 as a novel interacting protein of TCP10L. This mutual interaction relies on the LZ domains of both TCP10L and MAD1. TCP10L's transcriptional activity was reported shortly after its cloning (7). However, no potential DNA-binding motif was found in the structural analysis of the TCP10L peptide. Therefore, we proposed a hypothesis that TCP10L might interact with other transcriptional factors to exert its function. Two proteins, death-associated protein kinase 3 (DAPK3) and MAD4, have been reported to be TCP10L-interacting partners in our early studies $(10,14)$.

DAPK3 has been demonstrated to be a positive regulator in cell apoptosis (15). As a protein kinase, DAPK3 is able to phosphorylate itself as well as a variety of substrates, thereby participating in the regulation of cell apoptosis (16). Three nonsynonymous point mutations of DAPK3 were recently reported in various human tumor specimens, all of which rendered the tumor cells increased cell survival potential and resistance to chemotherapy (17). Given that TCP10L and DAPK3 could also form heterodimers in a LZ-dependent way, it is of interest to investigate whether TCP10L is also involved in DAPK3's proapoptotic function.

MAD4 belongs to the MAD family and inhibits c-Myc-dependent cell transformation in rat embryo cells (18). MAD4 was shown to interact with TCP10L by a yeast two-hybrid screen at the beginning of this TCP10L project (10). Later, judging that MAD4 and MAD1 shared significant homology in their structural domains, and also because MAD1 has been extensively studied during embryo development, cell transformation and cancer progression, we tested the possibility of a TCP10L-MAD1 interaction. In this study, we provided different pieces of evidence, including co-immunoprecipitation, GST-pull down and immunofluorescence staining. Our conclusion is that TCP10L interacts with MAD1. To date, MAD1 has been reported to exert diverse roles, including regulation of gene transcription and translation, cell apoptosis, cell cycle progression, cell differentiation and genome stability $(19,20)$. Consistently, 
we also observed that HCC cells over-expressing MAD1 exhibited suppressed gene transcription, G1 arrest and inhibited cell growth rate. More importantly, cells co-expressing MAD1 and TCP10L exhibited a remarkably enhanced suppressive effect on gene transcription, cell cycle progression and cell growth, which strongly suggests a synergistic relationship between TCP10L and MAD1. Additionally, the interaction-deficient mutant lost the synergistic function, indicating the mutual interaction is necessary for TCP10L to affect MAD1.

Recent studies revealed that the MAD1 protein has a very short half-life in cells, and its protein level is tightly regulated by different regulation mechanisms. Different cytokines and growth factors are able to induce the expression of MAD1 (21). Posttranslational modification, such as ubiquitination and phosphorylation, affects MAD1 protein stability. c-IAP1, a member of the inhibitor of apoptosis protein (IAP) family, is found to be a potential ubiquitin ligase for MAD1 (13). In addition, Ser-145 phosphorylation of MAD1 itself by $\mathrm{p} 90$ ribosomal kinases (RSK) and p70 S6 kinase (S6K) accelerates the ubiquitination and degradation of MAD1 (22). In our work, we reported a new mechanism for MAD1 regulation, which is that TCP10L could maintain the steady-state level of MAD1 protein in cells in a LZ domain-dependent manner. Interestingly, we also found TCP10L could interact with c-IAP1 (data not shown). It is therefore of great interest to identify whether TCP10L affects the ubiquitination of MAD1 in future studies. Previously, we have reported a down-regulated expression pattern of TCP10L in human HCC specimens (9). Together, these data lead to a hypothesis that during cancer progression down-regulated TCP10L results in MAD1 protein being more easily degraded by the proteasome, which then facilitates MYC-mediated cancer progression.

\section{MATERIALS AND METHODS}

\section{Cell culture, plasmid construction and siRNA transfection} HEK293T, HeLa, SMMC-7721 and SK-Hep1 cells were cultured in Dulbecco's Modified Eagle Medium (DMEM) supplemented with $10 \%(\mathrm{v} / \mathrm{v})$ Fetal Bovine Serum (FBS) at $37^{\circ} \mathrm{C}$ with $5 \% \mathrm{CO}_{2}$. Recombinant TCP10L plasmids and TCP10L mutant plasmids were described previously (8). The full-length MAD1 cDNA was amplified from a Marathon cDNA library (Clontech, USA) before being subcloned into pCMV-HA/pCMV-Myc vectors. MAD1 mutant constructs were generated by the KOD-Plus Mutagenesis Kit (TOYOBO, Japan). Two different siRNAs targeting TCP10L and control siRNA were synthesized by GenePharma, Inc. (Shanghai, P. R. China). Plasmids and siRNA oligos were transiently transfected into cells by using Lipofectamine 2000 reagent (Invitrogen, USA) according to the manufacturer's instructions.

\section{Immunoprecipitation assay}

Transfected HEK293T cells lysed $24 \mathrm{~h}$ post-transfection in BC100 buffer (Cell Signaling Technology, USA). The whole-cell lysates were immunoprecipitated with the monoclonal anti-Myc antibody-conjugated agarose beads (Sigma, USA) at $4^{\circ} \mathrm{C}$ overnight. The beads were washed five times with $\mathrm{BC} 100$ buffer and the precipitated proteins were subjected to Western blot. To carry out endogenous immunoprecipitation assays, HEK293T cells were lysed with BC100 buffer, and the cell lysates was centrifuged. The supernatant was precleared with protein A/G beads (Sigma, USA) and then incubated with anti-MAD1 antibody (Proteintech, USA) overnight. Thereafter, protein AVG beads were added, and the complexes were incubated at $4^{\circ} \mathrm{C}$. After $2 \mathrm{~h}$ of incubation, pellets were washed five times with $\mathrm{BC} 100$ buffer and resuspended in sample buffer and analyzed by SDS-PAGE.

\section{GST-pull down assay}

HEK293T cells overexpressing wildtype or mutant MAD1 were lysed at $36 \mathrm{~h}$ post-transfection with cell lysis buffer (Cell Signaling Technology, USA) at $4^{\circ} \mathrm{C}$. Cell lysates were centrifuged and the supernatant was precleared with glutathioneSepharose beads (Amersham Biosciences, USA). A similar amount of GST protein and GST-fusion protein was immobilized on glutathione-Sepharose beads. After washing with binding buffer (phosphate-buffered saline (PBS), $5 \mathrm{~mm}$ EGTA, $0.1 \%(\mathrm{v} / \mathrm{v})$ Triton $\mathrm{X}-100,0.5 \mathrm{~mm}$ PMSF), the beads were further incubated with precleared cell HEK293T lysates for $2 \mathrm{~h}$. The beads were then washed 5 times with binding buffer and resuspended in sample buffer before being subjected to SDS-PAGE analysis.

\section{Immunofluorescence and confocal microscopy}

HeLa cells were seeded on to sterile glass coverslips placed in 6 -well plates at a density of $3 \times 10^{5} /$ well. After transfection with indicated plasmids, cells were washed, fixed with $4 \%$ paraformaldehyde (Sigma, USA) at $37^{\circ} \mathrm{C}$ for $15 \mathrm{~min}$ and permeabilized with $0.2 \%$ TritonX-100 in PBS for 10 min. After fixation, cells were incubated with primary antibodies at $4^{\circ} \mathrm{C}$ overnight. Slides were washed, incubated with fluorescencetagged secondary antibodies (Alexa Fluor 568, Molecular probes, Invitrogen) and counterstained with DAPI (Vector Labs) for $1 \mathrm{~h}$ at $4^{\circ} \mathrm{C}$. Cells were visualized and imaged using a Zeiss LSM710 confocal microscope equipped with a $60 \times$ objective.

\section{Dual-Luciferase assay}

Briefly, HEK293T cells were plated into 24-well culture plates at a density of $1 \times 10^{5} /$ well and transfected with indicated plasmids. The pGL-myc-luc (Clontech, USA) reporter plasmid was co-transfected to investigate the transcriptional activity. The pRL-TK control vector was used for normalization. Cells were harvested at $24 \mathrm{~h}$ after transfection. Relative luciferase activity was determined using the Dual-Luciferase Reporter Assay System according to the manufacturer's instructions (Promega, USA). Experiments were repeated three times. The relative light units were measured by a luminometer (Promega, 
USA). Results are the representative of three independent experiments.

\section{Cycloheximide (CHX)-chase analysis}

HEK293T cells were transfected with MAD1 together with empty vector TCP10L or TCP10L- $\Delta \mathrm{LZ}$. At $24 \mathrm{~h}$ post transfection, cells were plated into 24-well culture plates at a density of $1 \times 10^{5} /$ well. Cells were cultured for another $24 \mathrm{~h}$ before $\mathrm{CHX}(100 \mu \mathrm{g} / \mathrm{ml})$ was added into the culture medium to inhibit protein synthesis. Cells were then collected at indicated time points and subjected to Western blot analysis.

\section{Statistical analysis}

Statistical analyses were performed using Student's two tailed t-tests. Values of $* \mathrm{P}<0.05, * * \mathrm{P}<0.01$ were considered statistically significant, and ns indicates not significant.

\section{ACKNOWLEDGEMENTS}

This work was supported by the National Natural Science Foundation of China (81472567) and 2012 “Chen Guang" project supported by Shanghai Municipal Education Commission and Shanghai Education Development Foundation.

\section{REFERENCES}

1. Luscher B (2012) MAD1 and its life as a MYC antagonist: an update. Eur J Cell Biol 91, 506-514

2. Sommer A, Bousset K, Kremmer E, Austen M and Luscher $B$ (1998) Identification and characterization of specific DNA-binding complexes containing members of the Myc/Max/Mad network of transcriptional regulators. J Biol Chem 273, 6632-6642

3. van Riggelen J, Yetil A and Felsher DW (2010) MYC as a regulator of ribosome biogenesis and protein synthesis. Nat Rev Cancer 10, 301-309

4. Cultraro CM, Bino T and Segal S (1997) Regulated expression and function of the c-Myc antagonist, Mad1, during a molecular switch from proliferation to differentiation. Curr Top Microbiol Immunol 224, 149-158

5. Cerni C, Skrzypek B, Popov N et al (2002) Repression of in vivo growth of Myc/Ras transformed tumor cells by Mad1. Oncogene 21, 447-459

6. Laherty CD, Yang WM, Sun JM, Davie JR, Seto E and Eisenman RN (1997) Histone deacetylases associated with the mSin3 corepressor mediate mad transcriptional repression. Cell 89, 349-356

7. Chen Z, Yu L, Wu H et al (2003) Identification of a novel liver-specific expressed gene, TCP10L, encoding a human leucine zipper protein with transcription inhibition activity. Hum Genet 48, 556-563

8. Zhong Z, Qiu J, Chen X et al (2008) Identification of TCP10L as primate-specific gene derived via segmental duplication and homodimerization of TCP10L through the leucine zipper motif. Mol Biol Rep 35, 171-178

9. Zuo J, Cai H, Wu Y et al TCP10L acts as a tumor suppressor by inhibiting cell proliferation in hepatocellular carcinoma. Biochem Biophys Res Commun 446, 61-67

10. Jiang DJ, Yu HX, Hexige SY et al (2004) Human liver specific transcriptional factor TCP10L binds to MAD4. J Biochem Mol Biol 37, 402-407

11. Wang Y, Toury R, Hauchecorne M and Balmain N (1997) Expression and subcellular localization of the Myc superfamily proteins: c-Myc, Max, Mad1 and Mxi1 in the epiphyseal plate cartilage chondrocytes of growing rats. Cell Mol Biol (Noisy-le-grand) 43, 175-188

12. Rottmann S and Luscher B (2006) The Mad side of the Max network: antagonizing the function of Myc and more. Curr Top Microbiol Immunol 302, 63-122

13. Xu L, Zhu J, Hu X et al (2007) c-IAP1 cooperates with Myc by acting as a ubiquitin ligase for Mad1. Mol Cell 28, 914-922

14. Yu H, Jiang D, Guo Z et al (2005) TCP10L is expressed specifically in spermatogenic cells and binds to death associated protein kinase-3. Int J Androl 28, 163-170

15. Kawai T, Matsumoto $M$, Takeda $K$, Sanjo $H$ and Akira $S$ (1998) ZIP kinase, a novel serine/threonine kinase which mediates apoptosis. Mol Cell Biol 18, 1642-1651

16. Komatsu S and Ikebe M (2004) ZIP kinase is responsible for the phosphorylation of myosin II and necessary for cell motility in mammalian fibroblasts. J Cell Biol 165, 243254

17. Brognard J, Zhang YW, Puto LA and Hunter T (2011) Cancer-associated loss-of-function mutations implicate DAPK3 as a tumor-suppressing kinase. Cancer Res 71, 3152-3161

18. Hurlin PJ, Queva C, Koskinen PJ et al (1996) Mad3 and Mad4: novel Max-interacting transcriptional repressors that suppress c-myc dependent transformation and are expressed during neural and epidermal differentiation. EMBO J 15, 2030

19. Xu D, Popov N, Hou M et al (2001) Switch from Myc/Max to Mad1/Max binding and decrease in histone acetylation at the telomerase reverse transcriptase promoter during differentiation of HL60 cells. Proc Natl Acad Sci U S A 98, 3826-3831

20. Gunes C, Lichtsteiner S, Vasserot AP and Englert C (2000) Expression of the hTERT gene is regulated at the level of transcriptional initiation and repressed by Mad1. Cancer Res 60, 2116-2121

21. Werner $S$, Beer HD, Mauch $C$ and Luscher B (2001) The Mad1 transcription factor is a novel target of activin and TGF-beta action in keratinocytes: possible role of Mad1 in wound repair and psoriasis. Oncogene 20, 7494-7504

22. Zhu J, Blenis J and Yuan J (2008) Activation of PI3K/Akt and MAPK pathways regulates Myc-mediated transcription by phosphorylating and promoting the degradation of Mad1. Proc Natl Acad Sci U S A 105, 6584-6589 